\title{
Simulation of charge reversal in salty environments: Giant overcharging?
}

\author{
O. Lenz ${ }^{1, \mathrm{a}}$ and C. Holm ${ }^{1,2, \mathrm{~b}}$ \\ 1 Frankfurt Institute for Advanced Studies, Johann-Wolfgang-Goethe-Universität, Frankfurt am Main, Germany \\ 2 Max-Planck-Institut für Polymerforschung, Mainz, Germany
}

Received 1 November 2007 and Received in final form 10 December 2007

Published online: 27 February 2008 - (C) EDP Sciences / Società Italiana di Fisica / Springer-Verlag 2008

\begin{abstract}
We have performed MD simulations of a highly charged colloid in a solution of 3:1 and additional 1:1 salt. The dependency of the colloid's inverted charge on the concentration of the additional 1:1 salt has been studied. Most theories predict, that the inverted charge increases when the concentration of monovalent salt grows, up to what is called giant overcharging, while experiments and simulational studies observe the opposite. Our simulations agree with the experimental findings and shed light onto the weaknesses of the theories.
\end{abstract}

PACS. 64.70.pv Colloids - 41.20.Cv Electrostatics; Poisson and Laplace equations, boundary-value problems - 87.10.Tf Molecular dynamics simulation

\section{Introduction}

In this work we study the phenomenon of overcharging on the basis of the simple restrictive primitive model. When a highly charged colloid (or macroion) is put into a solution that contains multivalent counterions, its charge can become overcompensated, such that the effective charge of the colloid-ion-complex becomes oppositely charged. In the literature, this phenomenon is called charge reversal, charge inversion or overcharging.

Experimentally, the phenomenon has been demonstrated by electrophoresis experiments, where a reversed mobility has been observed for charged colloids in a solution that contained multivalent ions (for a review, see [1]). Only recently, experiments were able to directly measure charge reversal in a system of a solution of multivalent salt at a charged wall $[2,3]$.

From a theoretical point of view, the standard PoissonBoltzmann theory is not able to predict charge inversion. A number of theories such as integral equations [4-7], fieldtheoretic approaches $[8,9]$, and others, see for example the references in $[10,11]$, have been put forward to describe this effect. Most popular amongst these are the simple one-component plasma (OCP) theories, which originated in the Wigner-Crystal theory by Shklovskii $[12,13]$, and have some descendants by Levin [14] and a recent one by Pianegonda et al. [15]. The basic idea of these theories is, that on the surface of the colloid, the multivalent

\footnotetext{
a e-mail: olenz@fias.uni-frankfurt.de

b e-mail: holm@fias.uni-frankfurt.de
}

ions form a Wigner crystal or at least a strongly correlated liquid. Therefore, the charge can be overcompensated by the multivalent ions. The rest of the system is considered in a Poisson-Boltzmann-like fashion. Although these theories are pretty simple, they give good predictions in many cases.

Simulations have confirmed the phenomenon of overcharging [16-20], and they are mostly consistent with the OCP theories in the strong-coupling regime, which is close to the Wigner-crystal $(T=0)$ limit. A notable exception is the work by Messina et al. [21], which shows that overcharging is also possible in situations that are very far from the strong-coupling limit. Here, the overcharging was attributed to mostly an entropy-driven effect.

Realistic and in particular biological systems, very often contain a significant amount of "normal" monovalent salt. Therefore it is interesting to see what happens to the charge reversal when 1:1 salt is added to the system.

Experimentally, with growing concentration of monovalent salt, the inverted charge seems to decrease [1]. In contrast to that, most OCP theories predict a monotonous increase of the inverted charge when more salt is added. In an extension to Shklovskii's original theory, Nguyen, Grosberg and Shklovskii $[22,23]$ even predict so-called "Giant overcharging" [22] for high concentrations of monovalent salt, where the effective charge is larger than the bare (negative) charge of the colloid. Levin's theory [14] predicts a more moderate growth, while Pianegonda, Barbosa and Levin [15] have a more detailed view: for low salt concentrations, an increase for growing salt concentrations is 
predicted, which reverses into a decrease for higher concentrations.

Not many simulations have been performed of that situation, however, all of them seem to support the experimental finding, that the amount of reversed charge decreases with increasing salt content. Tanaka and Grosberg have performed simulations of the electrophoresis of a colloid in salt solution in a regime of very high surface charge [20]. Martin-Molina et al. [24,25] simulate the overcharging at a planar wall in salty environment under more realistic conditions, which should be valid in the limit of large colloids. Diehl and Levin [26] have performed Monte Carlo simulations of a spherical colloid, where the $\zeta$-potential is measured.

This work complements these simulations by analyzing the case of a spherical colloid in salty environment in the experimental parameter regime, and comparing the results to the various assumptions made in the OCP theories.

\section{System}

The model system we are considering is a single, spherical, highly charged colloid in a solution of asymmetric $3: 1$ salt and additional 1:1 salt in a cubic box with periodic boundary conditions and a side length of $L=225.8 \AA$, which corresponds to a spherical cell with a radius $R_{0}=140 \AA$.

The simulation box contains four particle types that are defined via their diameter $d_{i}$ and their charge $q_{i}$ :

- 1 charged colloid $\left(Q_{0}=-300 e_{0}, d_{0}=100 \AA\right)$,

$-200(+3)$ counterions $\left(q_{(+3)}=3 e_{0}, d_{(+3)}=4 \AA\right)$,

$-\left(300+n_{\text {salt }}\right)(-1)$ coions $\left(q_{(-1)}=-1 e_{0}, d_{(-1)}=4 \AA\right)$,

$-n_{\text {salt }}(+1)$ counterions $\left(q_{(+1)}=1 e_{0}, d_{(+1)}=4 \AA\right)$.

The colloidal surface charge density is $\sigma_{s}=0.95 \mathrm{e}_{0} \mathrm{~nm}^{-2}=$ $0.152 \mathrm{Cm}^{-2}$, which is in the experimentally relevant regime. 200 trivalent counterions correspond to a concentration of $c_{(+3)}=30 \mathrm{mM}$. Up to $n_{\text {salt }}=1300$ monovalent salt ion pairs were added (concentration $c_{\text {salt }}=196 \mathrm{mM}$ ). The system is overall electro-neutral and the colloid is fixed in the center of the simulation box.

All particles interact via the Coulomb interaction

$$
V_{\text {coulomb }}(r)=\ell_{b} \frac{q_{i} q_{j}}{r}
$$

where the $q_{i}$ are the charges of the respective particle types and $\ell_{b}=7.1 \AA$ is the Bjerrum length in water at room temperature. Furthermore, the steric repulsion between the particles is modelled via the core interaction

$$
\begin{aligned}
& V_{\text {core }}(r)= \\
& \begin{cases}4 \varepsilon\left(\left(\frac{\sigma}{r-r_{\text {off }}}\right)^{12}-\left(\frac{\sigma}{r-r_{\text {off }}}\right)^{6}\right)+V_{\text {shift }}, & \text { if } r-r_{\text {off }}<r_{\text {cut }}, \\
0, & \text { otherwise. }\end{cases}
\end{aligned}
$$

This is the well-known shifted Lennard-Jones potential with an offset of $r_{\text {off }}$. To take into account only the repulsive part of the potential, we choose $r_{\text {cut }}=2^{1 / 6}$ and

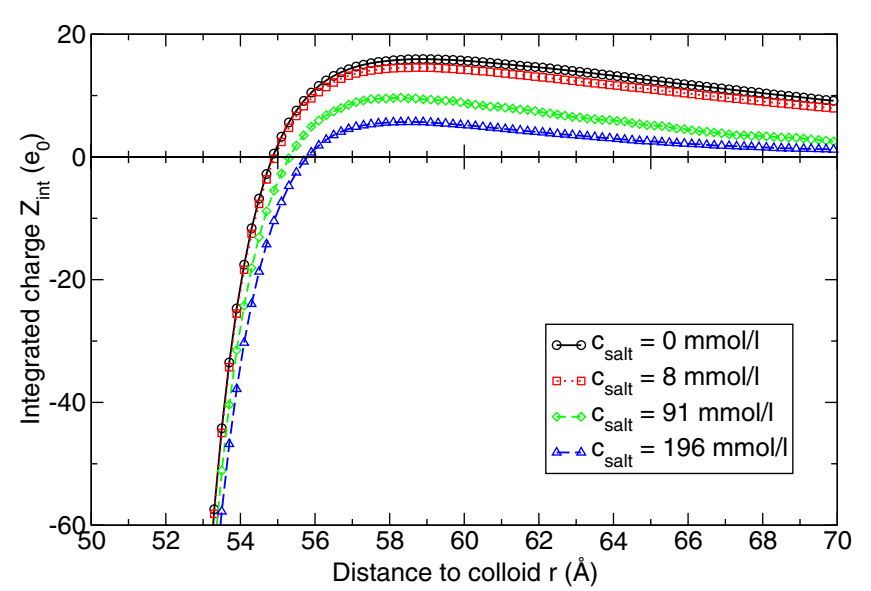

Fig. 1. Integrated charge $Z_{\text {int }}$ against the distance from the colloid center $r$ for different concentrations of additional salt $c_{\text {salt }}$.

$V_{\text {shift }}=\varepsilon$. Also, we choose $\sigma=1 \AA$ and $\varepsilon=1 \mathrm{kT}$ to ensure the same "hardness" of all interactions, so that the distance of closest approach is well defined. All particle sizes are modelled via the values of $r_{\text {off }}$ for the different interactions, which is defined by $r_{\text {off }}=\frac{1}{2}\left(d_{i}+d_{j}\right)-\sigma$, where $d_{i}$ and $d_{j}$ are the diameters of the respective particle types.

The system was simulated using a standard molecular dynamics (MD) algorithm using the Velocity Verlet algorithm in the $(\mathrm{N}, \mathrm{V}, \mathrm{T})$-ensemble, with an MD time-step of $\delta t=0.01$. To speed up the computation of the longranged electrostatic interactions, the P3M algorithm [27] was employed and tuned such that the maximal error in the forces was $\Delta_{\max }=0.001$. The water environment was modelled implicitly via the Langevin thermostat $(T=1$, $\gamma=0.5)$. Hydrodynamic interactions were neglected, as we are only interested in static-equilibrium observables.

All simulations were performed using the simulation package ESPResSo [28].

\section{Results}

Figure 1 shows a plot of the integrated charge $Z_{\text {int }}(r)$ against the distance to the center of the colloid for different concentrations of the additional salt $c_{\text {salt }}$, which is defined by

$$
Z_{\text {int }}(r)=Q_{0}+\sum_{i} \int_{0}^{r} q_{i} \rho_{i}\left(r^{\prime}\right) \mathrm{d} r^{\prime},
$$

where the $\rho_{i}(r)$ are the local densities of the different particle types in a distance $r$ to the center of the colloid. The plot clearly depicts, that in all simulations, the (negative) charge $Q_{0}$ of the colloid is overcompensated and has a maximum of up to $16 e_{0}$ at a distance of slightly more than two ion diameters from the colloidal surface.

In the following, we define the effective charge $Z_{\text {eff }}$ of the colloid as the maximum of the integrated charge distribution $Z_{\text {int }}$. This definition of the effective charge corresponds to the static model of Tanaka and Grosberg $[18,20]$. 


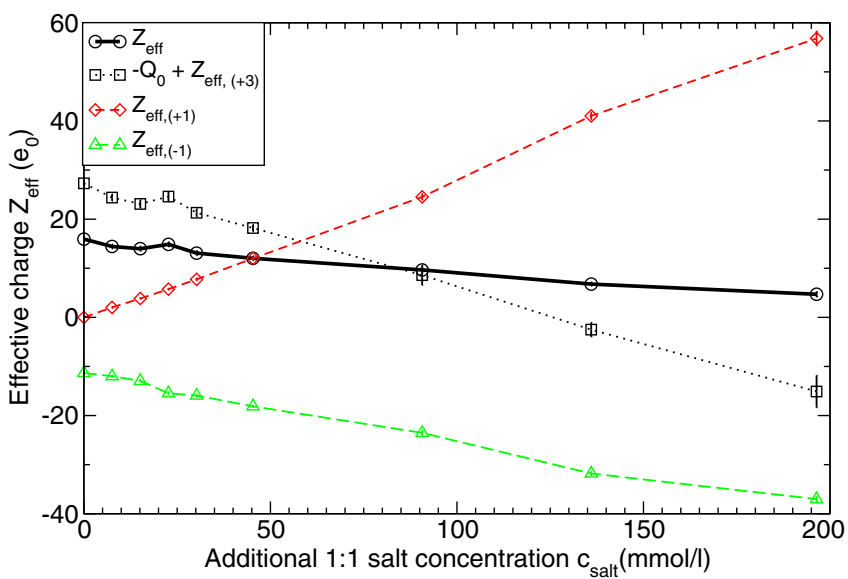

Fig. 2. Effective charge $Z_{\text {eff }}$ (maximum of the integrated charge $Z_{\text {int }}$ ) against the concentration of additional salt $c_{\text {salt }}$, and contributions of the different ion types.

Figure 2 contains a plot of $Z_{\text {eff }}$ and the contributions of the different ion types against the concentration $c_{\text {salt }}$ of additional monovalent salt. Details are revealed by the plots of the contributions of the different ion types in the figure. Keep in mind, that only by subtracting the bare charge $Q_{0}$ of the colloid from the contribution of the multivalent $(+3)$ ions $Z_{\text {eff. }(+3)}$, the different contributions are brought to the same scale. This stresses, that at all values of $c_{\text {salt }}$, the multivalent ions are responsible for the largest part of the compensation of the colloid's bare charge.

Note, that our definition of $Z_{\text {eff }}$ is not identical to the definition used in any of the previously cited theories, where only the multivalent ions contribute to the effective charge. To be able to directly compare with the theories, one can look at the plot of the quantity $-Q_{0}+Z_{\text {eff,(+3) }}$, i.e. the contribution of the multivalent ions $Z_{\mathrm{eff},(+3)} \mathrm{mi}-$ nus the bare charge $Q_{0}$ of the colloid. In contrast to what Shklosvkii's and Levin's theories predict, this quantity monotonously decreases with growing salt concentration. Also, it does not show a maximum, as the theory of Pianegonda et al. would predict [15].

However, it is also apparent, that the number of multivalent ions that contribute to $Z_{\text {eff }}$ decreases with growing concentration of additional salt, up to a point where the multivalent ions alone would not suffice to compensate $Q_{0}$ anymore. This is in contradiction to a fundamental assumption of the OCP theories, that we will discuss in the following section.

Instead, the contribution of monovalent $(+1)$ counterions $Z_{\text {eff,(+1) }}$ rapidly increases and is only partly compensated by the also increasing (negative) contribution of monovalent coions $Z_{\text {eff, }(-1)}$.

\subsection{Strongly correlated liquid but no screening}

All OCP theories predict, that the mutlivalent ions form a strongly correlated liquid on the colloidal surface, provided that the coupling is large enough. The plasma parameter of our systems is $\Gamma_{2 D}=\left(\pi \sigma_{s} \ell_{B} v^{3}\right)^{1 / 2}=6.3$, therefore we

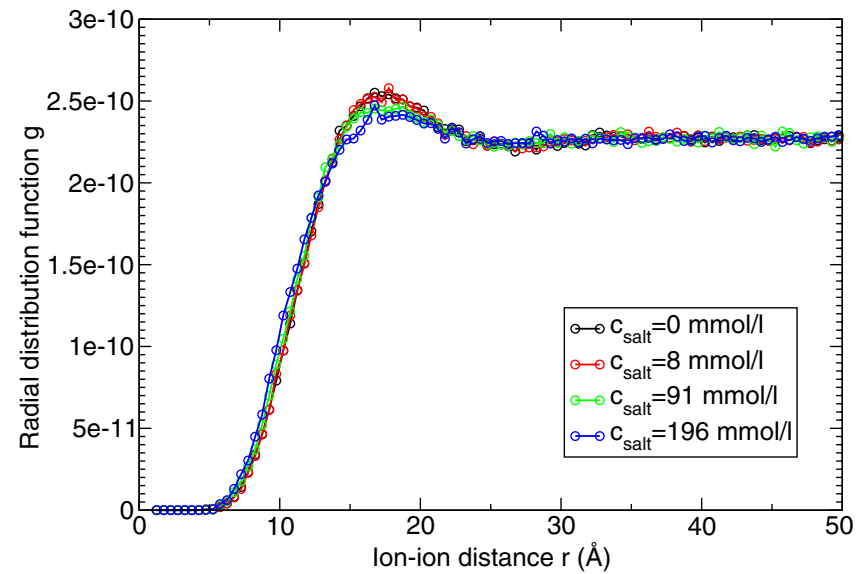

Fig. 3. Radial distribution function $g(r)$ of the trivalent counterions on the colloidal surface that contribute to the effective charge for different concentrations of additional 1:1 salt $c_{\text {salt }}$.

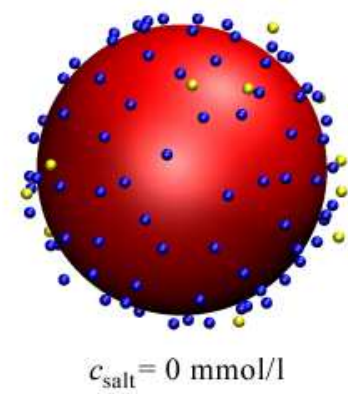

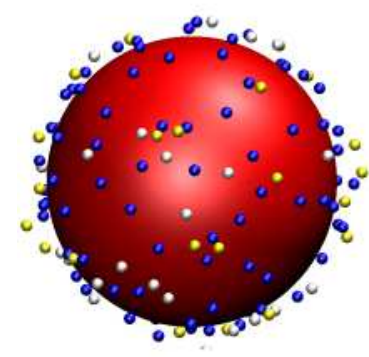

$c_{\text {salt }}=91 \mathrm{mmol} / 1$

•: $(+3)$-ions $\quad \cup:(+1)$-ions

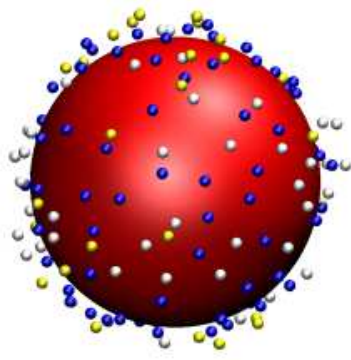

$c_{\text {salt }}=196 \mathrm{mmol} / 1$

$\bullet:(-1)$-ion
Fig. 4. Snapshots of the colloid and the ions at the surface that contribute to the effective charge $Z_{\text {eff }}$ for different concentrations of additional 1:1 salt $c_{\text {salt }}$.

are in the strong-coupling regime. This view is supported by the plot of the radial distribution function $g(r)$ of the trivalent ions that contribute to $Z_{\text {eff }}$ in Figure 3, which shows a correlation hole at very low distances and a (weak) peak at a distance of about $a=17 \AA$. Also, the simulation snapshots in Figure 4 show that the trivalent counterions indeed bind and completely cover the colloidal surface and form what looks like a liquid-like structure.

According to Shklosvkii's theory, the only effect of adding monovalent salt to the system should be to enhance the Debye-screening of the electrostatic interactions between the multivalent ions. This effect should be most 


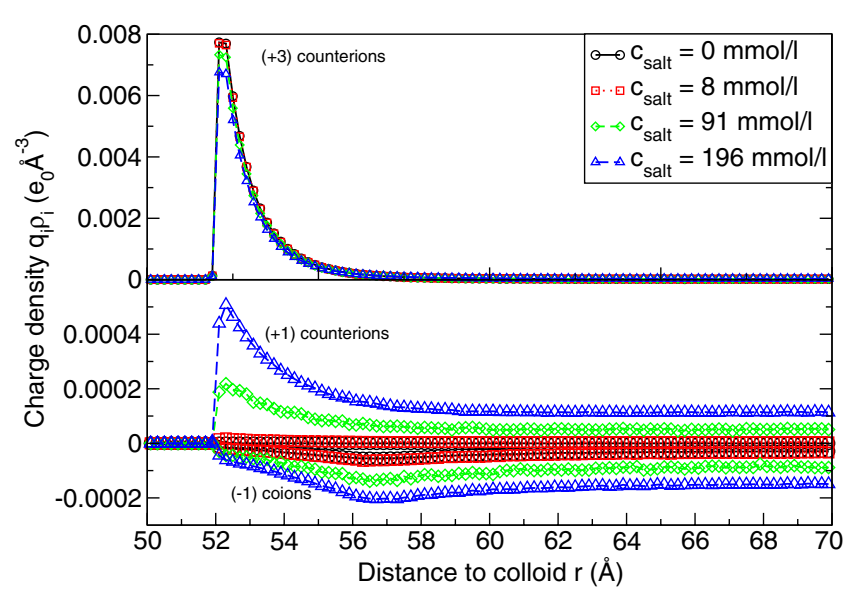

Fig. 5. Contribution of the different ion types to the charge density $\rho$ against the distance from the colloid center $r$ for different concentrations of additional salt $c_{\text {salt }}$. Note the different scales on the $y$-axis!

prominent, when the Debye screening length of the monovalent salt, which is defined by

$$
r_{S}=\left(8 \pi \ell_{B} c_{\text {salt }}\right)^{-1 / 2}
$$

is smaller than the preferred distance $a$ between the multivalent ions. In our simulations, we used values of the screening length of down to $r_{S}=8 \AA$, which is clearly smaller than the preferred distance $a$ between the trivalent ions. Still, $g(r)$ does not show a shift in the position of the first peak, which would be expected, if the preferred ion distance $a$ would be reduced. This refutes the idea, that Debye screening plays a significant role for the structure of the multivalent ions close to the surface.

Instead, we see a small decrease in the height of the peak of $g(r)$, meaning that the ions density decreases. This observation is consistent with the fact that less trivalent ions contribute to the effective charge at higher salt concentrations.

\subsection{Monovalent ions on the surface}

As was shown above, even though the contribution of the trivalent ions to the effective charge is most important, also the monovalent counter- and coions contribute to the effective charge $Z_{\text {eff }}$. Where are those ions located?

Figure 5 shows a plot of the charge densities $q_{i} \rho_{i}$ of the different ion types against the distance to the colloid center $r$. As was to be expected, with increasing $c_{\text {salt }}$, the density of monovalent ions close to the colloid increases. Interestingly, the maximum of the monovalent counterion density is at the same distance to the colloid as the maximum of the trivalent counterions for all salt concentrations. Note, however, that the maximum of the integrated charge $Z_{\text {int }}$ is at much larger values of $r \approx 60 \AA$.

While the value at the maximum of the density of the $(+1)$-ions increases with growing $c_{\text {salt }}$, the maximum value of the density of the multivalent ions decreases, i.e. some

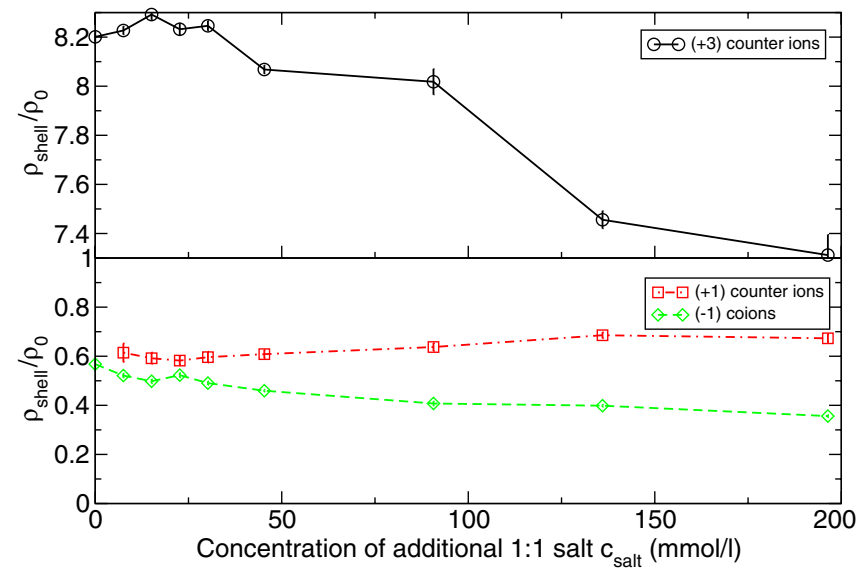

Fig. 6. Densities of the different ion types on the colloidal surface relative to the corresponding bulk densities $\rho_{\text {shell }, i} / \rho_{0, i}$ against the concentration of additional 1:1 salt $c_{\text {salt }}$.

of the multivalent counterions are replaced by monovalent counterions, which reduces the effective charge. This fact shows a weakness in the OCP theories, as these do not take into account that monovalent counterions play any role for the structure on the colloidal surface.

On the other hand, the monovalent coions form a weak, smeared-out second layer, that can also be seen as an indicator of the charge reversal of the colloid.

\subsection{Multivalent ions in the bulk}

Figure 6 shows a plot of the quantitity $\rho_{\text {shell,i }} / \rho_{0, i}$, where the $\rho_{0, i}=N_{i} / L^{3}$ are the bulk densities of the different ion types and the $\rho_{\text {shell,i }}$ are the respective densities of the ions in the shell that contributes to the effective charge. Not surprisingly, at $c_{\text {salt }}=0$, the density of the trivalent ions close to the colloid is much greater than the bulk density of the trivalent ions, while the density of the monovalent coions is smaller than in the bulk.

The value of $\rho_{\text {shell, }(+1)} / \rho_{0,(+1)}$ for the monovalent counterions is almost constant for increasing salt concentration $c_{\text {salt }}$, which indicates that the increasing contribution of monovalent $(+1)$ counterions to the effective charge is simply due to the growing bulk density of these ions.

On the other hand, the value of $\rho_{\text {shell,i }} / \rho_{0, i}$ of the $(+3)$ counterions and the $(-1)$-coions decreases when more salt is added. This shows, that with growing salt concentration, more and more of these ions prefer to stay in the bulk.

This can be interpreted in favour of the theory of Pianegonda et al. [15], who take into account that the (negative) solvation energy of the multivalent ions in the bulk is larger when more salt is there.

This effect reduces the reversed charge with growing salt concentration, which is also the reason why the theory of Pianegonda et al. is the only OCP theory that correctly describes a decrease in the effective charge at higher salt concentrations. 


\section{Conclusions}

To conclude, our simulations give support to the basic assumption of the OCP theories, namely that the overcompensation of the colloidal charge by multivalent ions is well described by a strongly correlated liquid. However, the theories have a few shortcomings when it comes to the description of the effect of additional monovalent salt.

In particular, the theories predict, that with growing salt concentration, the strongly correlated liquid can pack more densly on the colloidal surface, as the multivalent ions are screened by the monovalent salt. This assumption is not confirmed in our simulations. High salt concentrations seem not to influence the size of the Wigner-Seitz cell, and consequently, we do not see giant charge reversal. The giant overcharge observed in the parameter regime of Tanaka and Grosberg [18] is in our point of view due to the special choice of parameters. For the strong coupling regime considered, all ions form Bjerrum pairs, hence the concept of screening becomes meaningless.

Instead, with growing salt concentration, monovalent counterions are pushed onto the colloidal surface, and they replace some of the multivalent ions, which lowers the effective charge. This effect is not included into the current OCP theories.

Finally, we observed, that not only the trivalent counterions are pushed out of the region close to the surface of the colloid when more salt is added, but also some of the monovalent coions leave the region. This is consistent with the assumption of Pianegonda et al. [15], that the multivalent counterions and the monovalent coions can gain favourite solvation energy when more salt is in the bulk.

Therefore we conclude, that the theories of Shklovskii and Levin fail to describe the phenomenon of colloidal charge reversal under the influence of additional 1:1 salt correctly, as they are missing important concepts. On the other hand, the more recent theory of Pianegonda et al. [15] contains the necessary ingredients for a good description, even though the quantitative agreement with simulations is still not satisfactory [25].

The authors would like to thank Marcia C. Barbosa, Boris I. Shklovskii and Yan Levin for fruitful discussions, the PROBRAL project of the Deutscher Akademischer Austauschdienst (DAAD) for financial support of the exchange with M. Barbosa, and the Deutsche Forschungsgemeinschaft (DFG) for funding.

\section{References}

1. M. Quesada-Pérez, E. González-Tovar, A. Martín-Molina, M. Lozada-Cassou, R. Hidalgo-Alvarez, ChemPhysChem 4, 234 (2003).
2. K. Besteman, M.A.G. Zevenbergen, H.A. Heering, S.G. Lemay, Phys. Rev. Lett. 93, 170802 (2004).

3. K. Besteman, M.A.G. Zevenbergen, S.G. Lemay, Phys, Rev. E 72, 061501 (2005).

4. M. Lozada-Cassou, R. Saavedra-Barrera, D. Henderson, J. Chem. Phys. 77, 5150 (1982).

5. E. González-Tovar, M. Lozada-Cassou, J. Chem. Phys. 83, 361 (1985).

6. H. Greberg, R. Kjellander, J. Chem. Phys. 108, 2940 (1998).

7. R. Kjellander, "Distribution function theory of electrolytes and electrical double", in Electrostatic Effects in Soft Matter and Biophysics, edited by C. Holm, P. Kékicheff, R. Podgornik, NATO Sci. Ser. II, Vol. 46 (Kluwer Academic Publishers, Dordrecht, 2001) pp. 317-408.

8. R.R. Netz, H. Orland, Europhys. Lett. 45, 726 (1999).

9. A.G. Moreira, R.R. Netz, "Field-theoretic approaches to classical charged systems", in Electrostatic Effects in Soft Matter and Biophysics, edited by C. Holm, P. Kékicheff, R. Podgornik, NATO Sci. Ser. II, Vol. 46 (Kluwer Academic Publishers, Dordrecht, 2001).

10. R. Hidalgo-Álvarez, A. Martin, A. Fernandez, D. Bastos, F. Martinez, F.J. de las Nieves, Adv. Colloid Interface Sci. 67, 1 (1996).

11. B. Jönsson, H. Wennerström, "When ion-ion correlations are important in charged colloidal systems", in Electrostatic Effects in Soft Matter and Biophysics, edited by C. Holm, P. Kékicheff, R. Podgornik, NATO Sci. Ser. II, Vol. 46 (Kluwer Academic Publishers, Dordrecht, 2001).

12. V.I. Perel, B.I. Shklovskii, Physica A 274, 446 (1999).

13. B.I. Shklovskii, Phys. Rev. E 60, 5802 (1999).

14. Y. Levin, Rep. Prog. Phys. 65, 1577 (2002).

15. S. Pianegonda, M.C. Barbosa, Y. Levin, Europhys. Lett. 71, 831 (2005).

16. R. Messina, C. Holm, K. Kremer, Europhys. Lett. 51, 461 (2000).

17. R. Messina, C. Holm, K. Kremer, Eur. Phys. J. E. 4, 363 (2001).

18. M. Tanaka, A.Yu Grosberg, J. Chem. Phys. 115, 567 (2001).

19. M. Tanaka, A.Y. Grosberg, Eur. Phys. J. E 7, 371 (2002).

20. M. Tanaka, Phys. Rev. E 68, 061501 (2003).

21. R. Messina, E. González Tovar, M. Lozada-Cassou, C. Holm, Europhys. Lett. 60, 383 (2002).

22. T.T. Nguyen, A.Y. Grosberg, B.I. Shklovskii, Phys. Rev. Lett. 85, 1568 (2000).

23. A.Y. Grosberg, T.T. Nguyen, B.I. Shklovskii, Rev. Mod. Phys. 74, 329 (2002).

24. M. Quesada-Perez, A. Martin-Molina, R. Hidalgo-Alvarez, Langmuir 21, 9231 (2005).

25. A. Martín-Molina, J.A. Maroto-Centeno, R. HidalgoÁlvarez, M. Quesada-Pérez, J. Chem. Phys. 125, 144906 (2006).

26. A. Diehl, Y. Levin, J. Chem. Phys. 125, 054902 (2006).

27. R.W. Hockney, J.W. Eastwood, Computer Simulations using Particles (McGraw-Hill, New York, 1981).

28. H.J. Limbach, A. Arnold, B.A. Mann, C. Holm, Comput. Phys. Commun. 174, 704 (2006). 